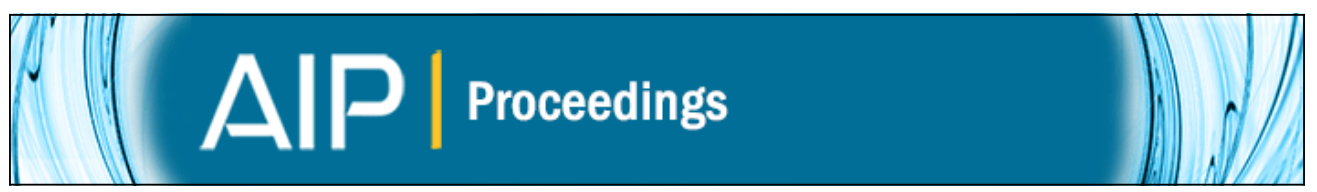

\title{
A Status Report on a Planet Search Around White Dwarf Stars
}

J. J. Hermes, F. Mullally, D. E. Winget, M. H. Montgomery, G. F. Miller, and J. L. Ellis

Citation: AIP Conference Proceedings 1273, 446 (2010); doi: 10.1063/1.3527860

View online: http://dx.doi.org/10.1063/1.3527860

View Table of Contents:

http://scitation.aip.org/content/aip/proceeding/aipcp/1273?ver=pdfcov

Published by the AIP Publishing

Articles you may be interested in

Searching for Planets with White Dwarf Pulsations: Spurious Detections

AIP Conf. Proc. 1331, 278 (2011); 10.1063/1.3556211

Latest Results from the DODO Survey: Imaging Planets around White Dwarfs AIP Conf. Proc. 1331, 271 (2011); 10.1063/1.3556210

Latest Results From The DODO Survey: Imaging Planets Around White Dwarfs AIP Conf. Proc. 1273, 440 (2010); 10.1063/1.3527859

Multi-Survey and Statistical Methods for Cool White Dwarf Discovery

AIP Conf. Proc. 1273, 180 (2010); 10.1063/1.3527799

Search for the coolest white dwarfs in the Galaxy

AIP Conf. Proc. 1273, 174 (2010); 10.1063/1.3527798 


\title{
A Status Report on a Planet Search Around White Dwarf Stars
}

\author{
J.J. Hermes*, F. Mullally ${ }^{\dagger}$, D.E. Winget*, M.H. Montgomery*, G.F. Miller* \\ and J.L. Ellis* \\ *Department of Astronomy and McDonald Observatory, University of Texas at Austin \\ ${ }^{\dagger}$ Department of Astrophysical Sciences, Princeton University
}

\begin{abstract}
We have continued monitoring a pilot sample of 15 isolated, pulsating DA white dwarfs for center-of-mass motion caused by a planetary companion. Roughly 7 years into our survey, we have preliminary evidence for periodic variations in pulse arrival times for at least two white dwarfs in our sample. The variations in these systems are unlikely to be caused by secular evolution and are possibly the result of motion of the white dwarf around a center of mass. We have yet to claim confirmation of a planet.

GD66 is a previously published candidate system, with a modulation in pulse arrival times that could be caused by a $2.0 M_{\mathrm{J}} \sin i$ planetary companion with an 8.3 year orbital period. Another candidate system, WD1354+0108, has a phase modulation consistent with a $0.7 M_{\mathrm{J}} \sin i$ planet at 2.3 AU (a 4.5 year orbit). We see similar behavior in two independent frequencies within this star, and while a sinusoid is currently a marginally better fit to the data than a straight line (as we might expect from cooling alone in a DAV), we are hesitant to over-interpret our results. Finally, we have a third system, WD0018+0031, that shows a change in pulse arrival times inconsistent with cooling alone; a $2.7 M_{\mathrm{J}}$ planet at an orbit of about $5 \mathrm{AU}$ could cause the observed trend. Observations of these candidate systems are ongoing in order to constrain any planetary companions that may be present.
\end{abstract}

Keywords: stars: white dwarfs — stars: oscillations — stars: planetary systems — stars: individual (GD66, WD1354+0108, WD0018+0031)

PACS: $97.20 . \mathrm{Rp} ;$ 97.10.Sj; 97.82.Fs

\section{INTRODUCTION}

A subset of pulsating white dwarfs exhibit extreme amplitude and frequency stability, making them reliable clocks. When such stable clocks have a planet orbiting them, their reflex motion around the center of mass of the system becomes measurable. The first confirmed success in the search for extrasolar planets came using such a timing method with pulsars [9], and this method has recently been used to identify a candidate planet around a pulsating compact object — a subdwarf B star [8].

The White Dwarf Group at the University of Texas at Austin has been monitoring a sample of 15 pulsating DAs since 2003, some even longer, for such behavior using Argos, a time-series photometer in use on the $2.1 \mathrm{~m}$ Otto Struve telescope at the McDonald Observatory [7]. These white dwarfs are typically classified as hot DAVs (hDAVs), as they fall near the blue edge of the ZZ Ceti instability strip and show extreme amplitude stability from year to year.

According to simple cooling theory [4], white dwarfs cool faster at hotter (more luminous) stages. Therefore, we expect that of all classes of pulsating white dwarfs, 
the DAVs are bound to show the slowest drift in period with time $(\dot{P}$, where $\mathrm{dP} / \mathrm{dt}=\dot{P})$. Kepler et al. 2005 [3] conclude that $\dot{P}$ is dominated by the rate of cooling alone for the DAV stars; they found that G117-B15A, an hDAV that has been monitored for more than 30 years, has a measured $\dot{P}=(3.57 \pm 0.82) \times 10^{-15} \mathrm{~s} \mathrm{~s}^{-1}$.

A companion produces reflex orbital motion of the DAV, causing a periodic variation in the pulse arrival times due to changing light-travel time. These variations will be superimposed on the evolutionary parabola, causing a modification to the $O-C$ equation [3]:

$$
O-C=\Delta E_{0}+\Delta P E+\frac{1}{2} P \dot{P} E^{2}+A \sin \left(\frac{2 \pi}{\Pi} t+\phi\right)
$$

The period of such a variations, $\Pi$, corresponds to the companion's orbital period, and we can use Kepler's third law to determine the companion's semi-major axis. Similarly, the amplitude, $A$, of the $O-C$ variations allows us to estimate the companion mass.

The DAV instability strip falls around $12,000 \mathrm{~K}$, so we expect cooling and thus the rate of period change to be slow. In fact, cooling alone should change the pulse arrival time by no more than $1 \mathrm{~s}$ over the 7 years of our pilot survey [1].

\section{CURRENT RESULTS: WD1354+0108}

WD1354+0108 is a low-amplitude pulsator in our sample; it is a $16.4^{\text {th }}$ magnitude DAV with a pulsation spectrum dominated by a 198.3 s periodicity with $0.6 \%$ amplitude. We have been able to isolate another stable periodicity at 291.6 seconds. The amplitude of the 198.3 s periodicity (black points in Fig. 1) is more than twice as high as the amplitude of the $291.6 \mathrm{~s}$ periodicity (red triangles), which explains why the errors in determining the time of pulse arrival are so much larger for the $291.6 \mathrm{~s}$ periodicity. This data has

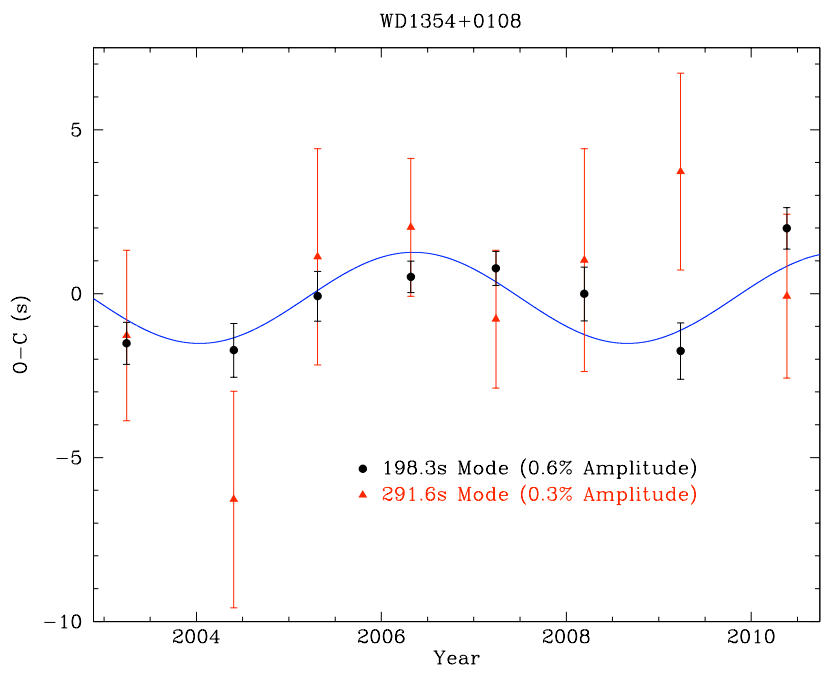

FIGURE 1: An $O-C$ diagram showing evidence of periodic behavior in pulse arrival times from the DAV WD1354+0108, which could be caused by an orbiting planet. We have been able to isolate two stable periods in the light curve, which are best-fit by a sinusoid that would corresponds to a $0.7 M_{\mathrm{J}} \sin i$ planet at 2.3 AU (a 4.6 year orbit). 
been welded together to the mid-point of each observing season to construct an $\mathrm{O}-\mathrm{C}$ diagram.

The best flat, parabolic fit to an $O-C$ diagram using both periodicities, as would be expected for evolution from cooling alone on a 7-year timescale, yields a $\dot{P}=(-1.94 \pm$ $1.73) \times 10^{-14} \mathrm{~s} \mathrm{~s}^{-1}$ with a $\chi_{\text {red }}^{2}=0.914$. The best sinusoidal fit to the data, on the other hand, yields a $\chi_{\text {red }}^{2}=0.862$. Therefore we claim that a sine curve fits the data marginally better than a straight-line fit. Such a sinusoid would corresponds to a $0.7 M_{\mathrm{J}} \sin i$ planet at 2.3 AU (a 4.6 year orbit).

We have also worked to characterize this $O-C$ diagram using a generalized LombScargle periodogram [10]. However, our data density is low, and while we see a visible peak in the periodogram corresponding to a 4.5 year period, it has yet to pass above a $1 \%$ false alarm probability threshold.

\section{GD66}

GD66 is a $15.6^{\text {th }}$ magnitude DAV with a rich pulsation spectrum, with at least 7 periodicities above $2 \mathrm{mma}$. GD66 was the most promising pulsating white dwarf hosting a planetary system in 2008 [5], and it remains a very persuasive candidate. Based on a mid-infrared search for planetary companions to white dwarfs with the Spitzer space telescope, a lack of infrared excess around $4.5 \mu \mathrm{m}$ suggests an upper limit of $5-7 M_{\mathrm{J}}$
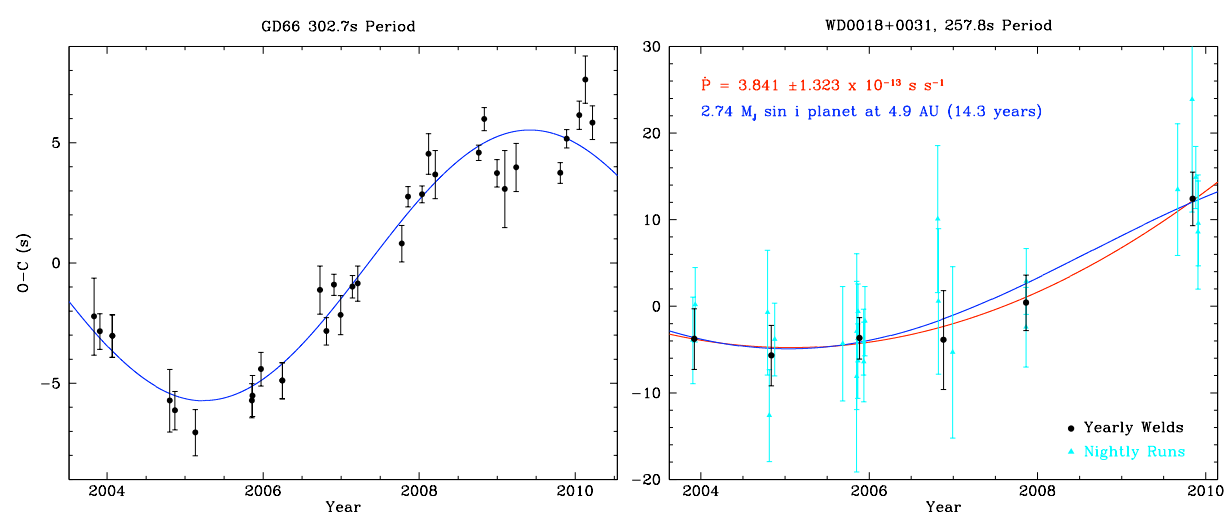

FIGURE 2. Left: An $O-C$ diagram of the 302.7 s periodicity in the DAV GD66. Cooling alone would modulate the phase by no more than 1 second over 7 years; the variations in the above diagram are best explained by reflex motion around the center of mass of the system. Right: An $O-C$ diagram of the highest-amplitude, $257.8 \mathrm{~s}$ periodicity present in the DAV WD0018+0031. The black points are welds of an entire year's worth of data; the smaller cyan triangles correspond to individual nights. The red, best-fit parabola corresponds to a secular evolution of $3.841 \pm 1.323 \times 10^{-13} \mathrm{~s} \mathrm{~s}^{-1}$, which is more than an order of magnitude more rapid than expected from cooling alone. The blue sinusoid corresponds to the effect a $2.7 M_{\mathrm{J}} \sin i$ planet at $4.9 \mathrm{AU}$ (a 14.3 year period orbit) might have on the DAV. We should be able to rule out one or the other within 2 years. 
on the mass of the candidate planet around GD66 [6]. Still, the 302.7 s, $1.1 \%$ amplitude periodicity in GD66 continues to produce an $\mathrm{O}-\mathrm{C}$ diagram inconsistent with evolution due to cooling (left panel of Fig. 2).

Cooling alone would modulate the phase of this mode in GD66 by no more than 1 second over 7 years. Thus, the observed variations are best explained by reflex motion around the center of mass of the system. Currently, the best-fit sinusoid to the data (plotted in blue) corresponds to a 2.0 $\mathrm{M}_{\mathrm{J}} \sin i$ planet at $3.5 \mathrm{AU}$ (an 8.3 year orbit).

\section{WD0018+0031}

WD0018+0031 is a $17.4^{\text {th }}$ magnitude DAV with low-amplitude pulsations, dominated by a 257.8 s periodicity with $0.6 \%$ amplitude. In the $O-C$ diagram (right panel of Fig. 2 ), cyan triangles correspond to individual nightly runs and black points correspond to welded, yearly observations. Again, from cooling alone, we expect pulse arrival times for a DAV to change by less than 1 s over 6 years, but here we are seeing a change of more than 10 seconds over such a span.

There are two good explanations for such behavior. Evolution internal to the star might be changing the period faster than we would expect from cooling. This would fit our observations in the form of the red parabola, which corresponds to a secular evolution of $3.841 \pm 1.323 \times 10^{-13} \mathrm{~s} \mathrm{~s}^{-1}$. Alternatively, a planetary companion might be moving the DAV around its center of mass. This would fit our data in the form of the blue sine curve, which could be produced by a $2.7 M_{\mathrm{J}} \sin i$ planet at $4.9 \mathrm{AU}$ (a 14.3 year orbit). We should be able to rule out one or the other by 2012 .

\section{ACKNOWLEDGMENTS}

This work is supported by the Norman Hackerman Advanced Research Program, under grant 003658-0255-2007, by a grant from the NASA Origins Program, NAG5-13094, and by the National Science Foundation, under grant AST-0909107.

\section{REFERENCES}

1. Bradley, P. A., Winget, D. E., \& Wood, M. A. 1992, ApJL, 391, L33

2. Kepler, S. O., et al. 1991, ApJL, 378, L45

3. Kepler, S. O., et al. 2005, ApJ, 634, 1311

4. Mestel, L. 1952, MNRAS, 112, 583

5. Mullally, F., Winget, D. E., De Gennaro, S., Jeffery, E., Thompson, S. E., Chandler, D., \& Kepler, S. O. 2008, ApJ, 676, 573

6. Mullally, F., Reach, W. T., De Gennaro, S., \& Burrows, A. 2009, ApJ, 694, 327

7. Nather, R. E., \& Mukadam, A. S. 2004, ApJ, 605, 846

8. Silvotti, R., et al. 2007, Nature, 449, 189

9. Wolszczan, A., \& Frail, D. A. 1992, Nature, 355, 145

10. Zechmeister, M., Kürster, M. 2009, A\&A, 496, 577 